\title{
Health-Related Quality of Life in Patients Treated with Continuous Ambulatory Peritoneal Dialysis and Automated Peritoneal Dialysis in Singapore
}

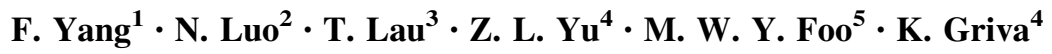

Published online: 14 July 2017

(c) The Author(s) 2017. This article is an open access publication

\begin{abstract}
Objective This study aimed to compare the health-related quality of life (HRQOL) in patients with end-stage renal disease (ESRD) treated with continuous ambulatory peritoneal dialysis (CAPD) and automated peritoneal dialysis (APD) in Singapore.

Methods The data used in this study were from two crosssectional surveys of ESRD patients. HRQOL was assessed using the Kidney Disease Quality of Life (KDQOL) instrument. Socio-demographic characteristics and clinical data were collected. The physical component summary (PCS) and mental component summary (MCS) scores, kidney disease component summary (KDCS) score and its three scales (symptoms, effects, burden), and one health utility score [EuroQol 5-dimension (EQ-5D)] were calculated and compared between CAPD and APD using multivariate linear regression.

Results In total, 266 patients were included, with 145 on CAPD (mean age 60.8 years) and 121 on APD (mean age 57.4 years). After adjustment for all variables collected, APD patients had significant higher scores in PCS and
\end{abstract}

K. Griva

psygk@nus.edu.sg

1 Manchester Centre for Health Economics, University of Manchester, Manchester, UK

2 Saw Swee Hock School of Public Health, National University of Singapore, Singapore, Singapore

3 Division of Nephrology, University Medicine Cluster, National University Health System, Singapore, Singapore

4 Department of Psychology, Faculty of Arts and Social Sciences, National University of Singapore, Singapore, Singapore

5 Department of Renal Medicine, Singapore General Hospital, Singapore, Singapore
KDQOL symptoms than CAPD patients, suggesting that APD was associated with better physical health and milder dialysis-related symptoms.

Conclusion The HRQOL of CAPD and APD patients was largely equivalent in Singapore, but APD patients seemed to experience better physical health and be less bothered by dialysis-related symptoms.

\section{Key Points for Decision Makers}

Peritoneal dialysis (PD) has been used as a practical and widespread alternative to conventional hemodialysis (HD) for end-stage renal disease (ESRD).

There are two forms of PD, continuous ambulatory PD (CAPD) and automated PD (APD). It has been shown that the clinical outcomes for these two PD modalities are comparable, so evidence on health-related quality of life (HRQOL) is important in guiding nephrologists and patients in their choice of PD modality.

The HRQOL of CAPD and APD patients was largely equivalent in Singapore, but APD patients seemed to experience better physical health and be less bothered by dialysis-related symptoms.

\section{Introduction}

Peritoneal dialysis (PD) has been used as a practical and widespread alternative to conventional hemodialysis (HD) for end-stage renal disease (ESRD) because of its 
advantages over HD, e.g., lower costs [1] and increased flexibility in lifestyle [2]. But it remains largely underutilized in most settings [3].

There are two forms of PD, continuous ambulatory PD (CAPD), which involves performing the PD exchanges manually, and automated PD (APD), which refers to all forms of PD using a mechanical device to assist the delivery and drainage of dialysate. It has been shown that the clinical outcomes for these two PD modalities are comparable $[4,5]$, so evidence on health-related quality of life (HRQOL) is important to contribute to guiding nephrologists and patients in their choice of PD modality. In the provision of healthcare, HRQOL is commonly evaluated, as it provides a good measure of treatment effectiveness by revealing how well an individual is functioning upon treatment [6]. For ESRD patients, HRQOL is an important predictor of clinical outcomes, and poor HRQOL could independently predict death and hospitalization of dialysis patients [7,8]. As the majority of old and frail patients undergoing dialysis are unlikely to receive kidney transplantation and would most likely remain in dialysis until the end of life, the importance of evaluating HRQOL is even more salient [9]. However, previous work of HRQOL outcomes for PD modalities has produced mixed evidence [10-12]. Furthermore, although there has been an exponential increase in HRQOL research in PD patients with Asian origin or in an Asian setting, most work has mainly compared PD with HD and has not been across PD modalities [2, 13, 14].

Therefore, this study aimed to evaluate HRQOL between PD modalities and to explore factors which could affect their HRQOL.

\section{Methods}

\subsection{Patients and Data}

Data used in this study were from two cross-sectional surveys, conducted between 2009 [15] and 2013 [16]. Participants were recruited from the PD center of Singapore General Hospital between 2009 and 2011 and from the renal center of the National University Hospital between 2012 and 2013. In both surveys, patients were approached by trained interviewers while awaiting consultation with a nephrologist. HRQOL data were collected using the kidney disease-specific HRQOL instrument Kidney Disease Quality of Life-Short Form (KDQOL-SF) in the first survey and its abridged version, the 36-item KDQOL (KDQOL-36), in the second survey, respectively. Socio-demographic characteristics were self-reported, and clinical data including co-morbidities measured using the Charlson Comorbidity Index (CCI), serum albumin and hemoglobin, dialysis vintage (i.e., time on dialysis), dependency status (i.e., self-care/assisted) and dialysis adequacy (i.e., Kt/V) were retrieved from medical records. Patients were included if they were aged $\geq 21$-year-old, on PD $\geq 3$ months, and able to communicate verbally and provide informed consent. This study was approved by the Institutional Review Board of the National University Health System, Singapore.

\subsection{Measures}

KDQOL-SF and KDQOL-36 are two commonly used instruments developed specifically for individuals with kidney disease and on dialysis [17]. Both instruments have been validated in ESRD patients in Singapore [18, 19]. KDQOL-SF includes Short Form-36 (SF-36) and 43 kidney disease-specific items; the KDQOL-36 contains a subset of the KDQOL-SF items, with Short Form-12 (SF-12) and 24 disease-specific items. Two summary scores, physical component summary (PCS) and mental component summary (MCS), can be calculated from SF-12, and the diseasespecific part generates three kidney disease-specific scales, i.e., symptoms, effects, and burden, and a kidney disease component summary (KDCS) score by averaging the three disease-specific subscales [20]. The EuroQol 5-dimension (EQ-5D) health utility score can be obtained from SF-12 using an established mapping function [21], and it has been demonstrated to be valid and sensitive in Singaporean dialysis patients [16]. For summary scores and health utility, higher scores represent better HRQOL, and for diseasespecific scales, higher scores represent fewer/milder symptoms, effects or burden due to kidney disease and dialysis.

\subsection{Statistical Analysis}

The socio-demographic and clinical characteristics were compared first, and then the three summary scores (PCS, MCS, and KDCS), three disease-specific scales and one health utility score (EQ-5D) were compared between patients with CAPD and those with APD. In the subsequent multivariate linear regression, all factor variables were coded into categorical variables in case the association was not linear and then entered into seven models, one for each of the HRQOL scores, regardless of their statistical significance. All analyses were performed using STATA 14.0, with $p<0.05$ being considered significant.

\section{Results}

A total of 266 patients were included, with 145 on CAPD and 121 on APD. Patients' mean [standard deviation (SD)] age was 59.3 (12.5) years, with $45.5 \%$ male, $74.4 \%$ 
Chinese, $80.8 \%$ having secondary or lower education, $71.1 \%$ married, and $88.3 \%$ living in a public residence. The mean (SD) CCI was 5.08 (1.67), and mean (SD) serum albumin and hemoglobin levels were 30.3 (5.6) $\mathrm{g} / \mathrm{l}$ and 10.9 (1.69) $\mathrm{g} / \mathrm{dl}$, respectively. For dialysis parameters, the mean (SD) dialysis vintage was 3.55 (3.28) years, and the mean (SD) $\mathrm{Kt} / \mathrm{V}$ value was 2.33 (0.88) per week.

No significant difference was observed in gender, ethnicity, marital status, housing type, co-morbidity, albumin level, hemoglobin level, and dialysis adequacy between CAPD and APD patients. But APD patients were younger than CAPD patients (mean age 57.4 vs. 60.8 years) and there were more individuals with high education, undergoing assisted dialysis, and with shorter dialysis vintage in the APD group. There was no significant difference in the QOL scores, with the exception that APD patients had higher KDQOL symptoms scores than CAPD patients (76.0 vs. 69.8). Full characteristics and QOL scores are shown in Table 1.

In multivariate analyses, APD was significantly associated with higher PCS and KDQOL symptoms scores, indicating patients had better physical health and milder dialysis-related symptoms (Table 2). We also found the following factors significantly associated with higher HRQOL scores (Table 2): young or old age, high albumin level, self-care dialysis and low dialysis adequacy.

\section{Discussion}

In view of the lack of clear evidence showing the advantage of one PD modality in clinical outcomes, a comparison of HRQOL between CAPD and APD patients would contribute to guiding the patient's choice and provide evidence for future cost-effectiveness assessment of PD treatments. Few studies have investigated this topic, and results are conflicting. De Wit et al. observed better mental health in APD patients [10], while Bro et al. found no difference in both physical and mental health [12]. Regarding the kidney disease-specific QOL, one previous study of incident PD patients showed that APD had advantages in KDQOL symptoms at 1 month, but significance disappeared at 12 months [22]. In our study, the HRQOL of CAPD and APD were almost equivalent, but physical health and KDQOL symptoms were in favor of APD.

The better physical health and fewer/milder symptoms of APD patients may be mainly explained by the nature of each PD modality. CAPD typically requires patients to manually perform exchanges of dialysate fluid four to five times a day, whereas APD is usually applied at night when the patient is asleep using an automated machine. The great time requirements of manual CAPD exchanges and additional abdominal weight due to dwelling dialysate in between the CAPD exchanges may be more likely to cause discomfort and more interruptions to daily activities, hence impacting HRQOL. In contrast, APD being an overnight procedure entails no dialysate weight bearing and allows more flexibility during the day for patients to pursue work, family and daily activities [23], which would be associated with better HRQOL. Also, compared to the manual exchanges in CAPD, the use of a dialysis machine in APD may lead to increased compliance with the prescribed PD regime and hence better disease management, which may in turn contribute to higher physical HRQOL. On the other hand, we could not rule out the possibility that the study participants may have been self-selected for better outcomes such as milder dialysis-related symptoms since our study cohort comprised prevalent PD patients with a mean dialysis vintage over 3 years. The health utility measured by the EQ-5D index showed a very small difference between CAPD and APD patients, suggesting that the relative cost-effectiveness of these two $\mathrm{PD}$ modalities in Singapore would be mainly determined by their survival outcomes and associated costs.

We also observed the impact of demographic, clinical and dialysis-related characteristics rather than PD modality itself on HRQOL. First, the impact of age on physical health was non-linear, with the middle-aged showing worse QOL, but old patients reporting comparable results to the young. A similar trend has been observed in previous studies [2, 24]. This may be due to the greater adaptation to chronic dialysis and old patients' lower expectations regarding their health. Second, high albumin level was associated with higher scores in PCS, MCS, KDQOL effects and health utility index. These associations make good sense from the clinical perspective. A low albumin may reflect malnutrition, and it is known to be strongly related to higher risk for mortality and morbidity in dialysis patients $[2,25]$. Thus, it would be expected to be associated with poorer HRQOL. Third, self-care patients reported better physical health and better health utility measured using EQ-5D, in line with the previous study [9]. This result was not surprising because patients having physical difficulties such as decreased vision and strength would be more likely to use assisted PD; however, the impact of these physical difficulties cannot be adjusted by the analyses of this study. Last, higher dialysis adequacy was associated with lower QOL scores, different from the previous study showing the positive correlation of $\mathrm{Kt} / \mathrm{V}$ and HRQOL [26]. In clinical practice, a dialysis adequacy target value is set to reduce the mortality risk [27], but to reach this target, patients might experience adverse effects and the increased amount of time needed to perform the exchanges is less acceptable to patients [27]. These factors might adversely affect QOL in PD patients. 
Table 1 Socio-demographic, clinical, dialysis characteristics and the HRQOL scores of the patients

\begin{tabular}{|c|c|c|c|c|}
\hline & Total $(n=266)$ & CAPD $(n=145)$ & $\operatorname{APD}(n=121)$ & $p$ value \\
\hline \multicolumn{5}{|l|}{ Socio-demographic } \\
\hline Age, mean (SD) & $59.3(12.5)$ & $60.8(11.4)$ & $57.4(13.6)$ & $0.03 *$ \\
\hline Young (45 years) & $33(12.4 \%)$ & $15(10.4 \%)$ & $18(14.9 \%)$ & 0.13 \\
\hline Middle-aged (45-60 years) & $90(33.8 \%)$ & $44(30.3 \%)$ & $46(38.0 \%)$ & \\
\hline Old ( $>60$ years) & $143(53.8 \%)$ & $86(59.3 \%)$ & $57(47.1 \%)$ & \\
\hline Gender & & & & 0.99 \\
\hline Male & $121(45.5 \%)$ & $66(45.5 \%)$ & $55(45.5 \%)$ & \\
\hline Female & $145(54.5 \%)$ & $79(54.5 \%)$ & $66(54.5 \%)$ & \\
\hline Ethnicity & & & & 0.76 \\
\hline Chinese & $198(74.4 \%)$ & $109(75.2 \%)$ & $89(73.6 \%)$ & \\
\hline Malay/Indian/others & $68(25.6 \%)$ & $36(24.8 \%)$ & $32(26.4 \%)$ & \\
\hline Educational level & & & & $0.02 *$ \\
\hline Low (no/primary/secondary) & $215(80.8 \%)$ & $125(86.2 \%)$ & $90(74.4 \%)$ & \\
\hline High (tertiary/above) & $51(19.2 \%)$ & $20(13.8 \%)$ & $31(25.6 \%)$ & \\
\hline Marital status & & & & 0.99 \\
\hline Married & $189(71.1 \%)$ & $103(71.0 \%)$ & $86(71.1 \%)$ & \\
\hline Other & $77(28.9 \%)$ & $42(29.0 \%)$ & $35(28.9 \%)$ & \\
\hline Housing type & & & & 0.13 \\
\hline Private residence & $31(11.7 \%)$ & $13(9.0 \%)$ & $18(14.9 \%)$ & \\
\hline Public residence & $235(88.3 \%)$ & $132(91.0 \%)$ & $103(85.1 \%)$ & \\
\hline \multicolumn{5}{|l|}{ Clinical } \\
\hline $\mathrm{CCI}$ & $5.08(1.67)$ & $5.19(1.48)$ & $4.95(1.87)$ & 0.24 \\
\hline Albumin $(\mathrm{g} / \mathrm{l})$ & $30.3(5.6)$ & $29.9(5.2)$ & $30.8(6.0)$ & 0.20 \\
\hline Hemoglobin (g/dl) & $10.9(1.69)$ & $10.9(1.67)$ & $11.0(1.71)$ & 0.78 \\
\hline \multicolumn{5}{|l|}{ Dialysis } \\
\hline Dependency status & & & & $<0.01 * *$ \\
\hline Self-care & $164(61.6 \%)$ & $102(70.3 \%)$ & $62(51.2 \%)$ & \\
\hline Assisted & $102(38.4 \%)$ & $43(29.7 \%)$ & $59(48.8 \%)$ & \\
\hline Dialysis vintage (years) & $3.55(3.28)$ & $4.50(3.68)$ & $2.42(2.26)$ & $<0.001 * * *$ \\
\hline \multicolumn{5}{|l|}{ Dialysis adequacy } \\
\hline $\mathrm{Kt} / \mathrm{V}$ (per week) & $2.33(0.88)$ & $2.28(0.72)$ & $2.40(1.04)$ & 0.26 \\
\hline \multicolumn{5}{|l|}{ QOL scores } \\
\hline PCS & $37.1(9.8)$ & $36.2(9.6)$ & $38.1(9.7)$ & 0.10 \\
\hline MCS & $46.6(11.1)$ & $46.7(11.2)$ & $46.4(11.1)$ & 0.80 \\
\hline KDCS & $58.7(18.0)$ & $57.6(19.0)$ & $60.0(16.7)$ & 0.29 \\
\hline Symptoms & $72.6(18.4)$ & $69.8(18.6)$ & $76.0(17.7)$ & $<0.01 * *$ \\
\hline Effects & $69.1(21.0)$ & $67.9(21.4)$ & $70.5(20.4)$ & 0.31 \\
\hline Burden & $34.4(26.9)$ & $35.2(27.8)$ & $33.5(25.8)$ & 0.62 \\
\hline EQ-5D & $0.59(0.21)$ & $0.58(0.21)$ & $0.60(0.22)$ & 0.35 \\
\hline
\end{tabular}

$A P D$ automated peritoneal dialysis, $C A P D$ continuous ambulatory peritoneal dialysis, $C C I$ Charlson Comorbidity Index, EQ-5D EuroQol 5-dimension, $H R Q O L$ health-related quality of life, KDCS kidney disease component summary, MCS mental component summary, PCS physical component summary, $Q O L$ quality of life, $S D$ standard deviation

$* p<0.05, * * p<0.01, * * * p<0.001$
This study has several limitations. First, the HRQOL data were from two different versions of the KDQOL, which may influence patients' responses due to context effect [28]. Second, analyses were based on cross- sectional data, and hence causal inferences cannot be made. Third, the EQ-5D was based on mapping, which is suboptimal compared to the direct use of a preferencebased measure. 
Table 2 Coefficients of the independent predictor variables for HRQOL scores in peritoneal dialysis patients

\begin{tabular}{|c|c|c|c|c|c|c|c|}
\hline \multirow[t]{3}{*}{ Independent variable } & \multicolumn{7}{|c|}{ Dependent variable } \\
\hline & \multicolumn{3}{|c|}{ Component summary score } & \multicolumn{3}{|c|}{ KDCS subscale } & \multirow{2}{*}{$\begin{array}{l}\text { Health utility } \\
\text { EQ-5D }\end{array}$} \\
\hline & PCS & MCS & KDCS & Symptoms & Effects & Burden & \\
\hline Young (45 years) & Ref. & & & & & & \\
\hline Middle-aged (45-60 years) & $-5.31 * *$ & 0.58 & -2.44 & -6.30 & -2.46 & 1.44 & -0.088 \\
\hline Old ( $>60$ years) & -2.81 & 2.69 & 4.58 & 0.95 & 6.97 & 5.83 & -0.022 \\
\hline Male & Ref. & & & & & & \\
\hline Female & 0.61 & -1.01 & 1.97 & 0.33 & 3.39 & 2.18 & -0.003 \\
\hline Chinese & Ref. & & & & & & \\
\hline Malay/Indians/others & -2.56 & 0.77 & -1.42 & -0.81 & -4.11 & 0.65 & -0.039 \\
\hline Low education (no/primary/secondary) & Ref. & & & & & & \\
\hline High education (tertiary/above) & -1.10 & 2.31 & -1.49 & -2.36 & -4.42 & 2.32 & 0.007 \\
\hline Non-married & Ref. & & & & & & \\
\hline Married & 1.23 & -1.61 & -2.90 & -1.38 & -3.81 & -3.50 & 0.002 \\
\hline Housing type, public residence & Ref. & & & & & & \\
\hline Housing type, private residence & 0.80 & 0.08 & 2.13 & 2.89 & -3.01 & 9.10 & 0.006 \\
\hline Low CCI $(<5)$ & Ref. & & & & & & \\
\hline High CCI $(\geq 5)$ & -0.95 & -1.32 & -5.75 & -2.28 & -4.59 & -10.4 & -0.029 \\
\hline Low albumin level $(<37 \mathrm{~g} / \mathrm{l})$ & Ref. & & & & & & \\
\hline High albumin level ( $\geq 37 \mathrm{~g} / \mathrm{l})$ & $5.75^{* *}$ & $4.90 *$ & 6.38 & 7.52 & $10.7 *$ & 0.90 & $0.146^{* *}$ \\
\hline Low hemoglobin level $(<11 \mathrm{~g} / \mathrm{dl})$ & Ref. & & & & & & \\
\hline High hemoglobin level $(\geq 11 \mathrm{~g} / \mathrm{dl})$ & 1.70 & 0.62 & 2.47 & 2.33 & 1.97 & 3.12 & 0.037 \\
\hline CAPD & Ref. & & & & & & \\
\hline APD & $2.81 *$ & -0.56 & 2.63 & $6.90 * *$ & 4.78 & -3.78 & 0.039 \\
\hline Dependency status, assisted & Ref. & & & & & & \\
\hline Dependency status, self-care & $5.12 * * *$ & -0.20 & 2.79 & 4.50 & 6.03 & -2.16 & $0.085 * *$ \\
\hline Short dialysis vintage ( $<3.5$ years) & Ref. & & & & & & \\
\hline Long dialysis vintage ( $\geq 3.5$ years) & 0.02 & 0.42 & -0.97 & -1.66 & 0.31 & -1.55 & 0.011 \\
\hline Low dialysis adequacy ( $<2.0 /$ week $)$ & Ref. & & & & & & \\
\hline High dialysis adequacy ( $\geq 2.0 /$ week $)$ & -1.63 & -2.74 & -1.69 & -0.85 & -2.53 & -1.69 & $-0.065^{*}$ \\
\hline Total $R^{2}$ & 0.17 & 0.05 & 0.06 & 0.09 & 0.08 & 0.04 & 0.15 \\
\hline
\end{tabular}

All $p$ values for a given independent variable are controlled for all other independent variables in the model

$A P D$ automated peritoneal dialysis, $C A P D$ continuous ambulatory peritoneal dialysis, $C C I$ Charlson Comorbidity Index, $E Q-5 D$ EuroQol 5-dimension, HRQOL health-related quality of life, KDCS kidney disease component summary, $M C S$ mental component summary, $P C S$ physical component summary

$* p<0.05, * * p<0.01, * * * p<0.001$

\section{Conclusion}

The HRQOL of CAPD and APD patients was largely equivalent in Singapore, but APD patients seemed to experience better physical health and be less bothered by dialysis-related symptoms. In the context of increasing advocacy for expanding PD utilization, more work is necessary to evaluate the outcomes of PD modalities to inform modality selection and guide healthcare resource allocation.
Author Contributions FY designed the study, analyzed the data and drafted the article. KG and NL contributed to study design and the critical revision of the article draft. TL and MF provided medical information. ZY helped with the original data collection. All authors read and approved the final manuscript.

\section{Compliance with Ethical Standards}

Funding No funding was received for this study.

Conflict of interest Fan Yang, Nan Luo, Titus Lau, Zhenli Yu, Marjorie Wai Yin Foo, and Konstadina Griva have no conflicts of interest. 
Data availability statement The data that support the findings of this study are available from the corresponding author upon reasonable request.

Open Access This article is distributed under the terms of the Creative Commons Attribution-NonCommercial 4.0 International License (http://creativecommons.org/licenses/by-nc/4.0/), which permits any noncommercial use, distribution, and reproduction in any medium, provided you give appropriate credit to the original author(s) and the source, provide a link to the Creative Commons license, and indicate if changes were made.

\section{References}

1. Baboolal K, McEwan P, Sondhi S, Spiewanowski P, Wechowski J, Wilson K. The cost of renal dialysis in a UK setting-a multicentre study. Nephrol Dial Transplant. 2008;23(6):1982-9.

2. Yang F, Griva K, Lau T, Vathsala A, Lee E, Ng HJ, Mooppil N, Foo M, Newman SP, Chia KS, Luo N. Health-related quality of life of Asian patients with end-stage renal disease (ESRD) in Singapore. Qual Life Res. 2015;24(9):2163-71.

3. Johansen KL. Choice of dialysis modality in the United States. Arch Intern Med. 2011;171(2):107-9.

4. Michels WM, Verduijn M, Boeschoten EW, Dekker FW, Krediet RT. Similar survival on automated peritoneal dialysis and continuous ambulatory peritoneal dialysis in a large prospective cohort. Clin J Am Soc Nephrol. 2009;4(5):943-9.

5. Cnossen TT, Usvyat L, Kotanko P, van der Sande FM, Kooman JP, Carter M, Leunissen KM, Levin NW. Comparison of outcomes on continuous ambulatory peritoneal dialysis versus automated peritoneal dialysis: results from a USA database. Perit Dial Int. 2011;31(6):679-84.

6. Jofre R, Lopez-Gomez JM, Valderrabano F. Quality of life for patient groups. Kidney Int. 2000;57:S121-30.

7. Lowrie EG, Curtin RB, LePain N, Schatell D. Medical outcomes study short form-36: a consistent and powerful predictor of morbidity and mortality in dialysis patients. Am J Kidney Dis. 2003;41(6):1286-92.

8. Mapes DL, Lopes AA, Satayathum S, McCullough KP, Goodkin DA, Locatelli F, Fukuhara S, Young EW, Kurokawa K, Saito A, Bommer J, Wolfe RA, Held PJ, Port FK. Health-related quality of life as a predictor of mortality and hospitalization: the Dialysis Outcomes and Practice Patterns Study (DOPPS). Kidney Int. 2003;64(1):339-49.

9. Griva K, Goh CS, Kang WC, Yu ZL, Chan MC, Wu SY, Krishnasamy T, Foo M. Quality of life and emotional distress in patients and burden in caregivers: a comparison between assisted peritoneal dialysis and self-care peritoneal dialysis. Qual Life Res. 2016;25(2):373-84.

10. de Wit GA, Merkus MP, Krediet RT, de Charro FT. A comparison of quality of life of patients on automated and continuous ambulatory peritoneal dialysis. Perit Dial Int. 2001;21(3):306-12.

11. Michels WM, van Dijk S, Verduijn M, le Cessie S, Boeschoten EW, Dekker FW, Krediet RT, Group NS. Quality of life in automated and continuous ambulatory peritoneal dialysis. Perit Dial Int. 2011;31(2):138-47.

12. Bro S, Bjorner JB, Tofte-Jensen P, Klem S, Almtoft B, Danielsen H, Meincke M, Friedberg M, Feldt-Rasmussen B. A prospective, randomized multicenter study comparing APD and CAPD treatment. Perit Dial Int. 1999;19(6):526-33.
13. Kim H, An JN, Kim DK, Kim MH, Kim YL, Park KS, Oh YK, Lim CS, Kim YS, Lee JP. Elderly peritoneal dialysis compared with elderly hemodialysis patients and younger peritoneal dialysis patients: competing risk analysis of a Korean Prospective cohort study. PLoS ONE. 2015;10(6):e0131393.

14. Chen JY, Wan EYF, Choi EPH, Chan AKC, Chan KHY, Tsang JPY, Lam CLK. The health-related quality of life of Chinese patients on hemodialysis and peritoneal dialysis. Patient. 2017; doi:10.1007/s40271-017-0256-6.

15. Griva K, Kang AW, Yu ZL, Mooppil NK, Foo M, Chan CM, Newman SP. Quality of life and emotional distress between patients on peritoneal dialysis versus community-based hemodialysis. Qual Life Res. 2014;23(1):57-66.

16. Yang F, Lau T, Lee E, Vathsala A, Chia KS, Luo N. Comparison of the preference-based EQ-5D-5L and SF-6D in patients with end-stage renal disease (ESRD). Eur $J$ Health Econ. 2015;16(9):1019-26.

17. Hays RD, Kallich JD, Mapes DL, Coons SJ, Carter WB. Development of the kidney disease quality of life (KDQOL) instrument. Qual Life Res. 1994;3(5):329-38.

18. Yang F, Wang VW, Joshi VD, Lau TW, Luo N. Validation of the English version of the Kidney Disease Quality of Life questionnaire (KDQOL-36) in haemodialysis patients in Singapore. Patient. 2013;6(2):135-41.

19. Cheung YB, Seow YY, Qu LM, Yee AC. Measurement properties of the Chinese Version of the Kidney Disease Quality of Life-Short Form (KDQOL-SF) in end-stage renal disease patients with poor prognosis in Singapore. J Pain Symptom Manage. 2012;44(6):923-32.

20. http://www.rand.org/health/surveys_tools/kdqol/faq.html. Accessed 15 Aug 2016.

21. Franks P, Lubetkin EI, Gold MR, Tancredi DJ, Jia H. Mapping the SF-12 to the EuroQol EQ-5D Index in a national US sample. Med Decis Making. 2004;24(3):247-54.

22. Jung HY, Jang HM, Kim YW, Cho S, Kim HY, Kim SH, Bang K, Kim HW, Lee SY, Jo SK, Lee J, Choi JY, Cho JH, Park SH, Kim CD, Kim YL, Group ES. Depressive symptoms, patient satisfaction, and quality of life over time in automated and continuous ambulatory peritoneal dialysis patients: a prospective multicenter propensitymatched study. Medicine (Baltimore). 2016;95(21):e3795.

23. Mizuno M, Suzuki Y, Sakata F, Ito Y. Which clinical conditions are most suitable for induction of automated peritoneal dialysis? Renal Replace Ther. 2016;2(1):46.

24. Rebollo P, Ortega F. New trends on health related quality of life assessment in end-stage renal disease patients. Int Urol Nephrol. 2002;33(1):195-202.

25. Lopes AA, Bragg-Gresham JL, Goodkin DA, Fukuhara S, Mapes DL, Young EW, Gillespie BW, Akizawa T, Greenwood RN, Andreucci VE, Akiba T, Held PJ, Port FK. Factors associated with health-related quality of life among hemodialysis patients in the DOPPS. Qual Life Res. 2007;16(4):545-57.

26. Chen YC, Hung KY, Kao TW, Tsai TJ, Chen WY. Relationship between dialysis adequacy and quality of life in long-term peritoneal dialysis patients. Perit Dial Int. 2000;20(5):534-40.

27. Chen JB, Lam KK, Su YJ, Lee WC, Cheng BC, Kuo CC, Wu CH, Lin E, Wang YC, Chen TC, Liao SC. Relationship between Kt/V urea-based dialysis adequacy and nutritional status and their effect on the components of the quality of life in incident peritoneal dialysis patients. BMC Nephrol. 2012;13:39.

28. Fayers Peter M, Machin D. Quality of life: the assessment, analysis and interpretation of patient-reported outcomes. 2nd ed. England: Wiley; 2007. 